\title{
Vortex-Antivortex Lattices in Superconducting Films with Magnetic Pinning Arrays
}

\author{
M. V. Milošević and F. M. Peeter ** \\ Departement Fysica, Universiteit Antwerpen (Campus Drie Eiken), \\ Universiteitsplein 1, B-2610 Antwerpen, Belgium
}

(Dated: August 14, 2018)

\begin{abstract}
Novel vortex structures are found when a thin superconducting film (SC) is covered with a lattice of out-of-plane magnetized magnetic dots (MDs). The stray magnetic field of the dots confines the vortices to the MD regions, surrounded by antivortices which "crystallize" into regular lattices. First and second order transitions are found as magnetic array is made sparser or MD-magnetization larger. For sparse MD-arrays fractional vortex-antivortex states are formed, where the crystalsymmetry is combined with a non-uniform "charge" distribution. We demonstrate that due to the (anti)vortices and the supercurrents induced by the MDs, the critical current of the sample actually increases if exposed to a homogeneous external magnetic field, contrary to conventional SC behavior.

PACS numbers: 74.78.-w, 74.25.Op, 74.25.Qt, 74.25.Dw.
\end{abstract}

The physics of vortex-antivortex pairs in superconductors and superfluids has been of general interest for a long time. For instance, such pairs are predicted to exist in thin superconducting films at finite temperatures due to thermal fluctuations [1]. Entropy considerations show that above the sharply defined Kosterlitz-Thouless transition temperature $T_{K T}$, these vortex pairs start to unbind, causing the appearance of a finite resistance. Recently it was found that symmetry-induced antivortices can be formed in mesoscopic superconducting polygons [2] in a certain parameter-range, such that the vortexantivortex configuration complies with the geometry of the polygon. In our recent work, we studied the vortex structure of a superconducting film with a single out-ofplane magnetized dot on top [3]. The total flux penetrating the superconductor equals zero, and vortices cannot form in isolation; vortices and antivortices nucleate in pairs. A shell vortex structure was observed, with a vortex nucleus surrounded by an antivortex core (the socalled "vortex-molecule") with size-magnetization controlled magic numbers. These vortex configurations resemble the ones of electron dimples on the surface of liquid helium, electrons in quantum dots, colloidal suspensions and dusty particles in complex plasmas [4].

In the present article, we report further consequences of this superconducting Wigner crystallization, in case when a regular array of magnetic particles is deposited on the superconducting film. Modern advances in microfabrication and characterization techniques $[\underline{5}$. have allowed an experimental realization of such $\mathrm{SC} /$ ferromagnet(FM) hybrid systems. Arrays of magnetic particles are potential devices for applying well-defined local magnetic fields, which modulate the order parameter in an underlying superconductor. Refs. [6, 7] (and references therein) have explored a plethora of physical effects, including matching effects with ordered pinning arrays, where additional pinning contributions arise due to the magnetic nature of the pinning centers.
Here, we investigate the superconducting state of a thin SC film with a square array of submicron cubic magnetic dots with perpendicular magnetization (Fig. 1). To ensure that MDs and SC are not electronically coupled, we assume a thin layer of insulating oxide between them. We consider cubic MDs, although most of the previous experimental work was done on thin FM structures. Making the magnetic dots thicker facilitates their magnetizing in the out-of-plane direction and eliminates the extreme peak structure in the stray field profile close to the dot edge. The general physical behavior of the SC drawn out in this article is immune to the MD-thickness.

The energy difference between the superconducting and the normal state, in units of $H_{c}^{2} / 4 \pi$, is

$$
\begin{aligned}
\Delta \mathcal{G}_{s / n}= & \int\left[-|\Psi|^{2}+\frac{1}{2}|\Psi|^{4}+\frac{1}{2}|(-i \nabla-\mathbf{A}) \Psi|^{2}\right. \\
& \left.+\kappa^{2}\left(\mathbf{H}-\mathbf{H}_{0}\right)^{2}\right] d V,
\end{aligned}
$$

where $\mathbf{H}_{0}$ denotes the applied magnetic field (for example, $\mathbf{H}_{0}=\mathbf{H}_{m d}$ if magnetic dots are the only field source). Eq. (11) is given in dimensionless form, where all distances are measured in units of the coherence length $\xi$, the vector potential $\mathbf{A}$ in $c \hbar / 2 e \xi$, the magnetic field

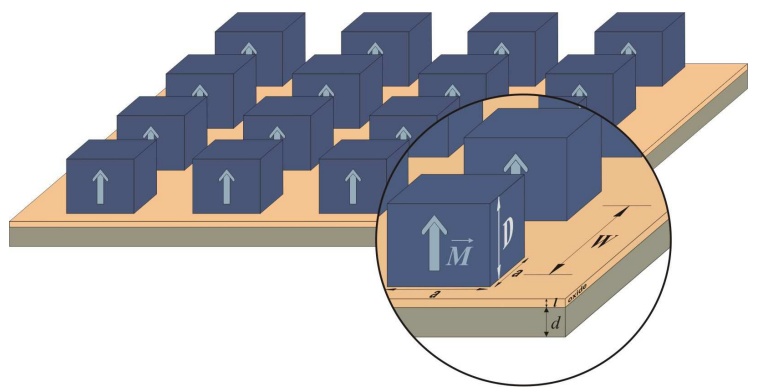

FIG. 1: Oblique view of the superconducting film and oxide layer (with thicknesses $d$ and $l$, respectively) underneath a regular array (with period $W$ ) of cubic magnetic dots. 
$\mathbf{H}$ in $H_{c 2}=c \hbar / 2 e \xi^{2}$, and the order parameter $\Psi$ in $\sqrt{-\alpha / \beta}$ with $\alpha, \beta$ being the GL coefficients. The minimization of Eq. (11) leads to well known GL equations which for thin superconductors $(d<\xi, \lambda)$ may be averaged over the SC thickness. We solve these two coupled equations, following a numerical approach proposed by Schweigert et al. (see Ref. 8] and references therein) on a uniform Cartesian grid with typically 10 points $/ \xi$ in each direction. In the present case, we took for the simulation region a rectangle $W_{x} \times W_{y}$, where $W_{x}=W_{y}=16 W$ (i.e. we simulate $16 \times 16$ supercell, see Fig. 1). Periodicity of the SC and the MD-lattice is included by applying periodic boundary conditions for $\mathbf{A}$ and $\Psi$ in the form $\mathbf{A}\left(\mathbf{r}+\mathbf{b}_{i}\right)=\mathbf{A}(\mathbf{r})+\nabla \eta_{i}(\mathbf{r})$, and $\Psi\left(\mathbf{r}+\mathbf{b}_{i}\right)=\Psi \exp \left(2 \pi i \eta_{i}(\mathbf{r}) / \Phi_{0}\right)[\underline{9}]$, where $\mathbf{b}_{i=x, y}$ are the supercell lattice vectors, and $\eta_{i}$ is the gauge potential. These boundary conditions mean that $\mathbf{A}, \Psi$ are invariant under lattice translations combined with specific gauge transformations. Since the vector potential of a regular array of magnetic dots is periodic by itself, we choose $\eta_{x}=\eta_{y}=0$. If the sample is exposed to an additional homogeneous perpendicular magnetic field $\mathbf{H}_{e x t}\left(\mathbf{H}_{0}=\mathbf{H}_{m d}+\mathbf{H}_{e x t}\right)$ we use the Landau gauge $\mathbf{A}_{e x t}=H_{e x t} x \mathbf{e}_{y}$ for the external vector potential and $\eta_{x}=H_{e x t} W_{x} y$ while $\eta_{y}=0$. Note that values of $H_{e x t}$ may not be chosen freely and must fulfill the flux quantization per supercell requirement following from the virial theorem [9].

To explore the superconducting state, we start from different (randomly generated) initial configurations, increase/decrease slowly ("sweep up/down") the magnetization of the MDs $M$ and let the vortex-configurationsolution relax to a steady-state one (in principle, it may be metastable). For given $M$, we recalculate the vortex structure of the film starting from: (i) the previously found configuration during the sweep, (ii) Meissner state ( $\Psi \approx 1)$ or (iii) the normal state $(\Psi \approx 0$ in the whole sample) as initial condition. By comparing the energies of all found vortex states we determine the ground state configuration. The obtained $M-W$ equilibrium vortex phase diagram is shown in Fig. 2 for the case of a SC film with thickness $d=0.2 \xi$ (this is, for example, an adequate value for a $50 \mathrm{~nm} \mathrm{~Pb}$ film at $T / T_{c}=0.97[$ [] $)$ and GL parameter $\kappa=1.2$ (approximately corresponding to the experimental values found for $\mathrm{Pb}, \mathrm{Nb}$, or $\mathrm{Al}$ films), covered with an oxide layer (thickness $l=0.1 \xi$ ) and an array of magnetic cubes with $a=D=2 \xi$ (see Fig. 1).

For small distance between MDs, the positive stray field under each magnetic dot is compensated by the negative fields of the neighboring dots, which decreases the amplitude of the magnetic field modulation seen by the $\mathrm{SC}$ (see Fig. 3). Note that the total flux through the $\mathrm{SC}$ is always zero. Since the demagnetizing factor of an infinite magnetic film is unity, for $W=a$ the magnetic field equals zero everywhere and the SC state exists for arbitrary value of $M$. Hence, the magnetization value at

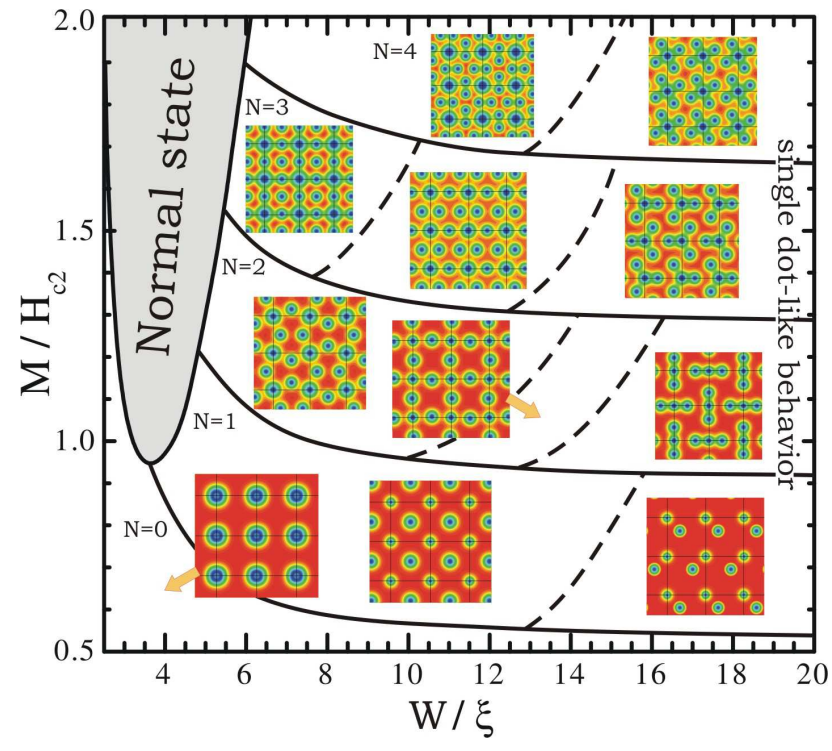

FIG. 2: Dependence of the vortex-antivortex configurations on the magnetization $(M)$ and period of the magnetic dot $(\mathrm{MD})$ lattice $(W)$. Solid lines denote transitions between states with different number of vortex-antivortex pairs per unit cell $(N)$, while dashed lines indicate second order configurational transformations for fixed $N$. The vortex structure is illustrated by the Cooper-pair density contourplots as insets (blue/red - low/high density), where the thin lines outline the unit cells of the MD lattice.

which the $\mathrm{S} / \mathrm{N}$ transition occurs decreases with increasing distance between the dots. In this region an unusual phenomenon occurs: increased strength (magnetization) of the dots drives the SC directly to the normal state, although the appearance of vortex-antivortex pairs is expected [3]. Namely, even if the critical conditions for their nucleation are achieved, there is not enough space for stabilizing antivortices in the narrow negative field areas. At the same time, the magnetic field under, and especially between the MDs is so large (see $W=3 \xi$ result in Fig. 3) that the SC state is suppressed globally.

When increasing $W$, the compensation effect diminishes, the field under MDs increases, suppressing locally the SC-state. Between the dots, negative stray field becomes lower (spread over wider area) and superconductivity survives. For small magnetization of the dots, the magnetic field is only able to suppress the superconducting order parameter under the edges of the MDs, where the induced currents are maximal [3]. Such MDs act as pinning centra for external vortices (e.g. resulting from the application of an external homogeneous magnetic field). This situation is very similar to the SC film perforated with a lattice of antidots. By increasing the magnetization, the negative flux between the MDs (as well as the positive one under the dots) increases, and the magnetic field lines can "join" into an antivortex (vortex). The critical magnetization and period $W$ for the first nucleated vortex-antivortex pair per 


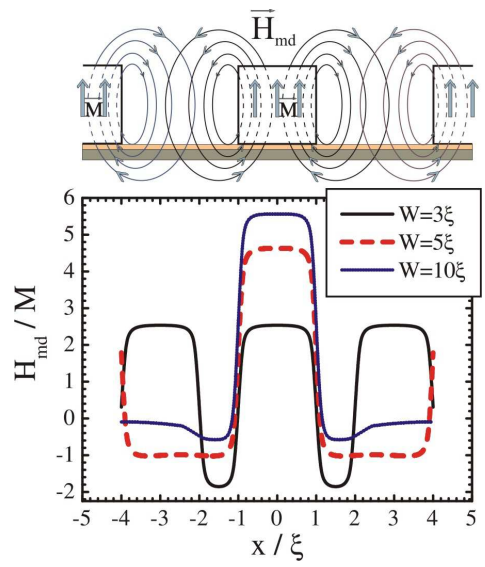

FIG. 3: Profile of the MD-magnetic field across the sample for different values of the period of the MD-lattice.

MD (in the ground state) are denoted in the lower part of Fig. 2 by a solid line. Using magnetostatic calculations, we determined that along this line, the positive flux under each MD is approximately constant, and equals $\Phi_{0 \mapsto 1}^{+} / \Phi_{0}=1.291 \pm 0.012$. We found that the additional positive magnetic flux necessary for the nucleation of the next vortex-antivortex pairs is quantized: $\Delta \Phi^{+} / \Phi_{0}=1.07 \pm 0.01$. These values of threshold fluxes weakly depend on the parameters of the SC, but strongly on the properties of the stray magnetic field determined by the geometrical dimensions of the MDs (i.e. $\Phi_{0 \mapsto 1}^{+}$ increases significantly with the size of the $\operatorname{dots} a$ ).

For dense lattices, the antivortices are compressed into narrow interstitial channels, forced to form regular and consequently rigid lattices, where now antivortices are "shared" by the neighboring MDs. The rigidness, together with the uniform distribution of vortices and antivortices, makes these ordered vortex structures resemble ionic crystals. In addition, one finds significant similarities in the physical mechanisms of crystallization. The somewhat simplified theory of cohesion in the ionic (and molecular) crystals assumes that the cohesive energy is entirely given by the potential energy of classical particles localized at equilibrium positions. Because the particles in ionic crystals are electrically charged ions, the main term in the interaction energy is the interionic Coulomb interaction. The other contribution comes from the strong short-range core-core repulsion due to the Pauli principle, without which the crystal would collapse. Analogy with our system follows from the present relations - namely, vortices and antivortices interact analogously to ions, except for the absent core-core repulsion, necessary for crystallization. This stabilizing factor is brought in our sample by the presence of magnetic dots, which effectively keep the vortices and surrounding antivortices apart. Therefore, each antivortex interacts with a magnetic dot coupled with the vortex underneath through a Lennard-Jones-like potential, form- ing superconducting ion-pairs, which when brought closer together form a two-dimensional ionic crystal. This fascinating parallel is best illustrated by the vortex-antivortex $N=1$ lattice from Fig. 2, which corresponds to the ionconfiguration on the surface of a $\mathrm{NaCl}$ crystal (and many other salts and oxides, e.g. $\mathrm{AgBr}, \mathrm{PbS}, \mathrm{FeO}$, etc.).

In $N=2$ crystal (each dot creates a double vortex and two antivortices), antivortex dimers are shared between the neighboring dots in such a way that each MD is surrounded by 4 antivortices arranged in a cross. For small $W$, the adjacent crosses are tilted with respect to each other. The tilt angle changes with $W$ (or magnetization $M$, see dashed lines in Fig. 2) and the configuration transforms through a second-order phase transition to square symmetry (tilt angle zero). This bipartite crystal now consists of two sublattices (of vortices and antivortices), where sites belonging to one lattice are connected only to the sites of another (see, e.g., the surface of the $\mathrm{ReO}_{3}$ crystal). In $N=3$ lattice, the orientational degree of freedom is lost, since antivortices crystallize in a perfect square lattice. With increasing distance between the MDs, the crystallization mechanism becomes more influenced by the inter-(anti)vortex interaction than the imposed symmetry, and the square lattice gradually transforms into a hexagonal one.

However, if the MDs are set further apart, the antivortex lattice bonds gradually break, leading to oriented clusters (rather than a crystal) of vortex-antivortex molecules around each MD, like in the case of a single magnetic dot on top of a SC [3]. For example, in $N=1$ state, the antivortices are no longer in the central interstitial position, but are bound to a particular MD. Their relative position is such that it maximizes the distance between them. The $N=3$ molecules have a specific orientational order in which molecules under adjacent columns of MDs are rotated by $60^{\circ}$, due to the repulsion of neighboring antivortex-trimers. Note that the distribution of antivortices in this case does not obey the symmetry of the magnetic potential (cubic MDs). This leads to an interesting scenario, where some of the vortex-antivortex pairs may annihilate in order to preserve the square symmetry of the vortex state at the expense of the energy, allowing the vortex configuration to crystallize again. In such manner, fractional states are formed, where some MDs "share" a vortex-antivortex pair (the number of pairs per $\operatorname{dot} N$ becomes a rational number). In Fig. 4 we show the Cooper-pair density plots of two typical fractional states for the square magnetic lattice $N=2^{1} / 2$ and $N=2^{3} / 4$. Here two species of vortices are present (doubly and triply charged), causing the adequate rearrangement of singly charged interstitial antivortices. Although with higher energy than the ground-state (for example, $\Delta E \approx 278 k T_{c}$ for $50 \mathrm{~nm} \mathrm{Nb}$ or $\mathrm{Pb}$ films at temperatures far from $T_{c}$ ), these states are metastable and experimentally observable.

The predicted new vortex configurations can be ob- 


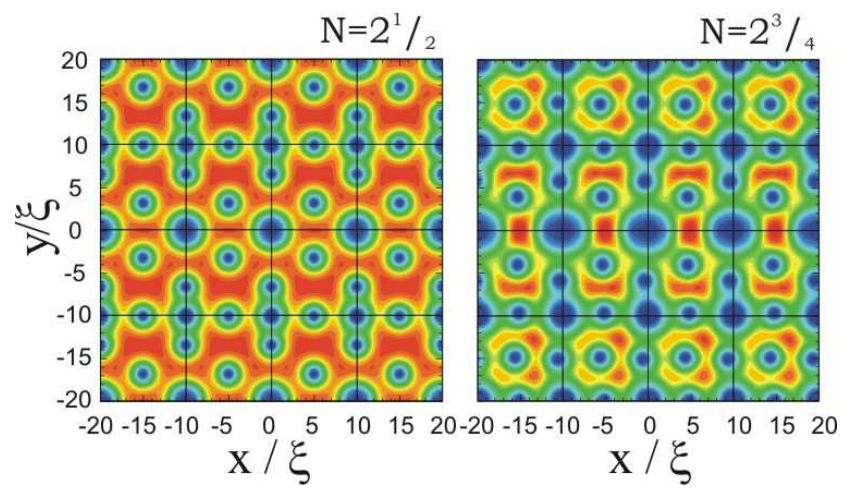

FIG. 4: The $|\Psi|^{2}$ contourplots of typical fractional vortexantivortex crystals for the $4 \times 4$ unit cell simulation region.

served experimentally by using e.g. scanning probe techniques like Hall and Magnetic Force Microscopy. These vortex-antivortex structures will strongly influence the pinning properties and the $\mathrm{SC}$ phase diagram. If one adds a homogeneous external magnetic field, such that the number of additional flux quanta matches the number of antivortices at the interstitial sites, one expects that annihilation occurs, resulting in a well known matching vortex configuration with all vortices pinned by the MDs. This leads to a peak in the critical current, as a function of the applied external field. This mechanism explains the recently observed phenomenon of magneticfield-induced superconductivity [10. In order to verify this, we exposed our sample with $W / \xi=6.25$ to a homogeneous magnetic field corresponding to the first matching field (one vortex per unit cell) and changed gradually the magnetization $M$, starting each time from the normal state. Then we apply current in the $x$ direction as $A_{c x}=$ const. (now $\mathbf{A}_{0}=\mathbf{A}_{m d}+\mathbf{A}_{e x t}+\mathbf{A}_{c}$ ) which does not interfere with our boundary conditions. When the critical value of $A_{c x}$ is exceeded, the motion of (anti)vortices can no longer be prevented and superconductivity is destroyed. The results of our calculations for the critical current $j_{c}$ as a function of the magnetization of the MDs are shown in Fig. 5 for the case with and without applied first matching field. If no external field is present (dark dots in Fig. 5), higher magnetization $M$ induces larger screening currents and $j_{c}$ monotonously decreases. The appearance of vortex-antivortex pairs decreases the total current in the sample through the phasechange contribution to the current. This leads to somewhat enhanced critical current which decreases further with magnetization and tends to zero. On the contrary, in the case of the first matching field (open dots in Fig. 5) the critical current equals zero if no pinning is present. With increasing magnetization, the antivortex-like currents [3] are increased, compensating the current of external vortices pinned by the dots. For $M / H_{c 2}=0.418$ maximal compensation is reached, resulting in the maximal critical current. With further increased magneti-

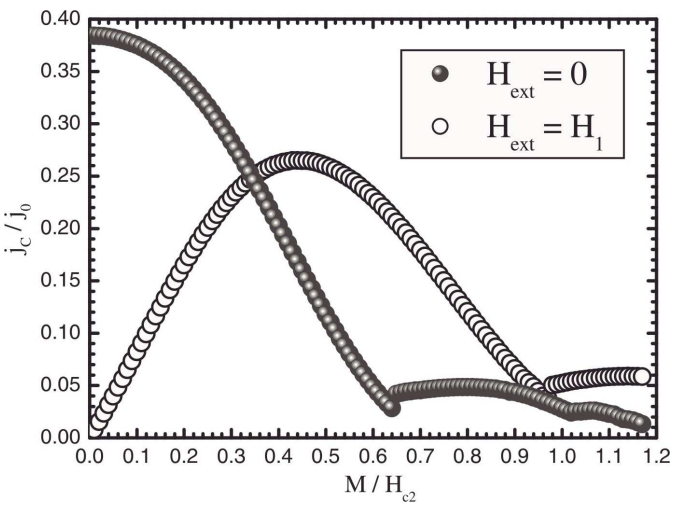

FIG. 5: Critical current (in units of $j_{0}=c H_{c 2} \xi / 4 \pi \lambda^{2}$ ) versus the magnetization of the magnetic dot lattice $(a / \xi=2$, $W / \xi=6.25)$ in the case of no applied external magnetic field $H_{\text {ext }}$ (dark dots) and for the first matching field (open dots).

zation, the qualitative behavior of $j_{c}$ is similar to the $H_{\text {ext }}=0$ case. Nevertheless, if an external magnetic field is present, the critical current of the sample for given $M$ is found to be actually higher. This demonstrates that, contrary to conventional superconductors, the superconductivity in SC-FM heterostructures is effectively enhanced by an applied magnetic field.

The authors acknowledge D. Vodolazov for valuable discussions. This work was supported by the Flemish Science Foundation (FWO-Vl), The Belgian Science Policy, the University of Antwerp (GOA), and the ESF programme "VORTEX".

Note added in proof: Very recently, Priour and Fertig [Phys. Rev. Lett. 93, 057003 (2004)] studied a similar system where an extremely thin SC and MDs were in immediate vicinity. This leads to much sharper magnetic field profiles, enhancing disorder in the mixed state.

* Electronic address: francois.peeters@ua.ac.be

[1] J.M. Kosterlitz and D.J. Thouless, J. Phys. C: Solid State Phys. 6, 1181 (1973); M.R. Beasley, J.E. Mooij, and T.P. Orlando, Phys. Rev. Lett. 42, 1165 (1979).

[2] L.F. Chibotaru et al., Nature (London) 408, 833 (2000); ibid. Phys. Rev. Lett. 86, 1323 (2001); V.R. Misko et al., Phys. Rev. Lett. 90, 147003 (2003).

[3] M.V. Milošević and F.M. Peeters, Phys. Rev. B 68, 024509 (2003).

[4] V.M. Bedanov and F.M. Peeters, Phys. Rev. B 49, 2667 (1994).

[5] J.I. Martin et al., Phys. Rev. Lett. 79, 1929 (1997).

[6] J.I. Martin et al., Phys. Rev. Lett. 83, 1022 (1999).

[7] M.J. Van Bael et al., Phys. Rev. B 59, 14674 (1999).

[8] V.A. Schweigert, F.M. Peeters, and P.S. Deo, Phys. Rev. Lett. 81, 2783 (1998).

[9] M.M. Doria, J.E. Gubernatis, and D. Rainer, Phys. Rev. B 39, 9573 (1989).

[10] M. Lange et al., Phys. Rev. Lett. 90, 197006 (2003). 